\section{Willie Sutton Was Right: It's Time to Turn to the Synovium to Drive Rheumatoid Arthritis Therapy}

Treatments for rheumatoid arthritis (RA) have come a long way in the past 20 years. Fifteen years of widespread use of methotrexate generated a paradigm shift in the management of RA; the advent of biologic response modifiers launched the next big shift. Coupled with early, aggressive therapy, these agents have made it possible to look beyond merely controlling symptoms to a treatment landscape where remission is now a reasonable possibility for nearly $50 \%$ of our patients with early disease ${ }^{1}$. At the same time, challenges remain for appropriately using available therapies. As effective as these agents are, they do not work for all patients, and despite years of searching, we still do not have systemic biomarkers that can reliably identify those patients who will respond. Combined with the tremendous costs of these drugs, this uncertainty means that RA treatment in 2016 remains an expensive, empiric proposition. We believe that it is time for the next paradigm shift, one that does not involve new therapies, but precision, targeted application of existing and future treatments. Willie Sutton was right ${ }^{2}$. And while much work remains to be done, we believe that it will soon be time to move beyond the bloodstream and look for guidance to the most critical tissue in this disease, the synovium.

One needs to look no further than the world of oncology to see the path that we must begin to take in rheumatology. In just a generation, oncologists have moved from the use of broad-based chemotherapeutic agents to personalized genetic profiling that allows them in many cases to identify the specific agent or agents most likely to effectively treat the patient's malignancy. Cancer therapy has moved from a disease-guided approach to a pathology-guided approach, then in turn to a molecular-guided approach, in which specific molecular alterations can drive the choice of therapy ${ }^{3}$. Admittedly, this transition has been easier because cancer is a genomic disease, in which somatic mutations can be identified as drivers of the pathology. Nevertheless, we think it is time to begin considering this type of profiling in
RA, a disease that also has a clear genomic component. Genome-wide association studies (GWAS), along with twin studies, have clearly shown a defined HLA component, more specifically the shared epitope, as a central contributor to $\mathrm{RA}^{4,5}$. While GWAS also uncovered a large number of genes that are linked to RA, the OR are small. Nonetheless, epigenetic modifications are now thought to be a major factor in the development of RA, and these alterations are all occurring at the cellular level in the synovium ${ }^{6,7}$.

What needs to happen to make molecular-guided therapy a reality in RA? Work by Dr. Paul Tak and colleagues used arthroscopic synovial biopsy prior to and following therapy ${ }^{8,9,10}$. Although such studies yield substantial amounts of synovial tissue, they are invasive, require surgical suites, and are expensive. Thus, this approach is not commonly practiced in the United States. Synovectomy and joint replacement surgery are other common mechanisms for researchers to obtain synovial tissue. However, these patients typically exhibit endstage disease characteristics and do not reflect the overall pathophysiology at the time when therapeutic decisions are made, prior to progressive joint damage. We believe that there are 3 important components that must be achieved to overcome the obstacles to using synovial tissue to guide therapy.

First, acquisition of adequate tissue for analysis needs to be something that can be done routinely for all newly diagnosed patients with RA, and for patients with existing disease for whom therapeutic changes are being considered. In this issue of The Journal, Najm and colleagues discuss their success in performing ultrasound (US)-guided synovial biopsies, heretofore a research tool, as part of standard clinical practice ${ }^{11}$.

Second, the sequencing techniques necessary for profiling synovial tissue must be sufficiently developed, widely available, and cost-effective enough for routine clinical use. As we will discuss below, the field has not yet reached this point, but we are not far off.

See US-guided synovial biopsy, page 2113

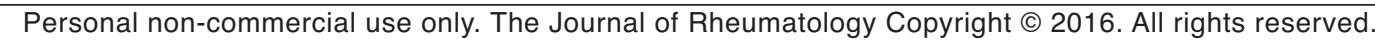


Finally, we will need to identify profiles that can indicate which patients will, or will not, respond to individual therapies. This remains the greatest challenge, but we believe it is time for our field to expand the search for these markers, even as we perfect the techniques that will make this type of analysis available for all patients with RA.

Regarding the acquisition of tissue, Najm and colleagues describe their success over an 8-year period with an US-guided biopsy technique using a Tru-cut manual biopsy needle ${ }^{11}$. During this time, they performed 76 biopsies in 74 patients, successfully retrieving histologically confirmed synovial tissue in 62 instances (81.6\%). Synovial lining tissue was identified in $92.6 \%$ of successful biopsies. Although the histological examination or PCR analysis yielded a definitive diagnosis in only $16.2 \%$ of the cases, and despite the unremarkable findings in this tissue using these relatively blunt analyses, the very fact that Najm, et al were able to retrieve synovial tissue in the vast majority of cases is the important observation. Importantly, the morbidity associated with the biopsy procedure was quite low, with only a single hemarthrosis, and no other complications reported during a mean followup of nearly 3 years. This study not only confirms the previous works that have used US-guided synovial biopsies, but extends their findings and provides support for general use in the clinic $^{8,12,13,14,15}$.

Musculoskeletal US has become an integral part of rheumatology practice, for both identification of synovitis and assisting with injections and arthrocentesis. It is a relatively small step from this approach, which has been widely adopted, to the use of US to acquire synovial biopsies. The learning curve is not steep, and the equipment and facilities needed for the biopsies are not much more extensive than those already used for US-guided arthrocentesis. In our own practice, our rheumatologist (AM), a certified ultrasonographer, was trained in the biopsy procedure ${ }^{14}$ and was able to perform 15 biopsies in just 11 months.

Once synovial biopsy tissue has been obtained, the next hurdle will be the widespread availability of techniques for analyzing this tissue at the molecular level. Currently, there is great interest in using synovial tissue for histological classification of patients. However, these types of studies do not allow for a comprehensive understanding of the molecular signatures that define not only the synovial tissue, but also the individual cells that form the tissue. Whole-tissue transcriptional profiling can be performed, but data from this type of study may be clouded by the fact that synovial tissue is extremely heterogeneous, so that distinct, but small, populations of cells may be overwhelmed by larger populations. With as little as 4 pieces of synovial biopsy material weighing less than $4 \mathrm{mg}$, it is now possible to disaggregate the tissue into as many as 70,000 individual cells, upon which flow cytometry can be used to sort individual cell populations, or even single cells. Whole-population or single-cell transcriptomic studies can then be performed on these cells to give an unbiased transcriptional profile that may be indicative of therapeutic response. As additional methodology improves, one can envision a fully comprehensive transcriptional (ChIP-seq, ATAC-seq) or proteomic approach by CyTOF (time-of-flight cytometry) to be used on these precious samples.

The final, necessary step to make synovial biopsies clinically useful will be availability of data on the molecular findings associated with response to individual therapies. Systemic biomarkers have proven relatively ineffective at predicting response to biologic therapies ${ }^{16}$. Synovium, however, remains the target tissue for these therapies, and seems likely to have the highest probability of yielding biomarkers predicting clinical response. Specific histological findings in the synovium, such as persistent macrophages in the synovial sublining ${ }^{17}$, have already been shown to be broad indicators of response to therapy. The next step will be to pinpoint more specific predictive synovial biomarkers, which will likely be found at the cellular and molecular level. Collaborative work is already under way to try to identify these markers ${ }^{18,19}$.

As the treatment of RA has progressed, the rheumatology community has embraced the treat-to-target approach, using objective clinical measures of response to define the need to escalate therapy in order to achieve the best outcomes. At the same time, we lack information on exactly which therapies to select for each patient. While we have begun to see comparative effectiveness trials, which can address therapeutic options at the population level, the value of these trials in identifying the right therapy at an individual level is limited. As the world moves into the era of personalized medicine, we remain mired in a trial-and-error approach to RA treatment. A true personalized approach to therapy will require us to move beyond clinical phenotypes, and even systemic biomarkers, as indicators of the likelihood of response. We see the synovium, and the precise molecular characterization of the cells it comprises, as the most hopeful way forward to truly individualized care. The work of Najm and colleagues demonstrates that this synovium can be effectively acquired in the course of clinical practice, and not just in a research setting. Once this approach is coupled with the ongoing advances in molecular profiling, we look forward to the day, in the not too-distant future, when we will be able to define the specific therapy best suited to each individual patient.

ERIC M. RUDERMAN, MD,

Division of Rheumatology,

Northwestern University Feinberg School of Medicine;

ARTHUR M. MANDELIN, MD, PhD,

Division of Rheumatology,

Northwestern University Feinberg School of Medicine;

HARRIS R. PERLMAN, PhD,

Division of Rheumatology,

Northwestern University Feinberg School of Medicine, Chicago, Illinois, USA.

Personal non-commercial use only. The Journal of Rheumatology Copyright @ 2016. All rights reserved. 
Address correspondence to Dr. E.M. Ruderman, 675 North St. Clair, Suite 14-100, Chicago, Illinois 60611, USA;

E-mail: e-ruderman@northwestern.edu

\section{REFERENCES}

1. Ma MH, Scott IC, Kingsley GH, Scott DL. Remission in early rheumatoid arthritis. J Rheumatol 2010;37:1444-53.

2. Definition of Sutton's law. [Internet. Accessed October 12, 2016.] Available from www.emedicinehealth.com/script/main/art.asp?articlekey $=39253$

3. Zeron-Medina J, Ochoa de Olza M, Brana I, Rodon J. The personalization of therapy: molecular profiling technologies and their application. Semin Oncol 2015;42:775-87.

4. Gregersen PK, Silver J, Winchester RJ. The shared epitope hypothesis. An approach to understanding the molecular genetics of susceptibility to rheumatoid arthritis. Arthritis Rheum 1987;30:1205-13.

5. Goronzy J, Weyand CM, Fathman CG. Shared T cell recognition sites on human histocompatibility leukocyte antigen class II molecules of patients with seropositive rheumatoid arthritis. J Clin Invest 1986;77:1042-9.

6. Bottini N, Firestein GS. Epigenetics in rheumatoid arthritis: a primer for rheumatologists. Curr Rheumatol Rep 2013;15:372.

7. Frank-Bertoncelj M, Gay S. The epigenome of synovial fibroblasts: an underestimated therapeutic target in rheumatoid arthritis. Arthritis Res Ther 2014;16:117.

8. Gerlag DM, Tak PP. How to perform and analyse synovial biopsies. Best Pract Res Clin Rheumatol 2013;27:195-207.

9. Haringman JJ, Vinkenoog M, Gerlag DM, Smeets TJ, Zwinderman AH, Tak PP. Reliability of computerized image analysis for the evaluation of serial synovial biopsies in randomized controlled trials in rheumatoid arthritis. Arthritis Res Ther 2005;7:R862-7.

10. Kraan MC, Reece RJ, Smeets TJ, Veale DJ, Emery P, Tak PP. Comparison of synovial tissues from the knee joints and the small joints of rheumatoid arthritis patients: implications for pathogenesis and evaluation of treatment. Arthritis Rheum 2002;46:2034-8.

11. Najm A, Orr C, Heymann MF, Bart G, Veale DJ, Le Goff B. Success rate and utility of ultrasound-guided synovial biopsies in clinical practice. J Rheumatol 2016;43:2113-9.
12. Humby F, Kelly S, Bugatti S, Manzo A, Filer A, Mahto A, et al. Evaluation of minimally invasive, ultrasound-guided synovial biopsy techniques by the OMERACT filter-determining validation requirements. J Rheumatol 2016;43:208-13.

13. Humby F, Kelly S, Hands R, Rocher V, DiCicco M, Ng N, et al. Use of ultrasound-guided small joint biopsy to evaluate the histopathologic response to rheumatoid arthritis therapy: recommendations for application to clinical trials. Arthritis Rheumatol 2015;67:2601-10.

14. Kelly S, Humby F, Filer A, Ng N, Di Cicco M, Hands RE, et al. Ultrasound-guided synovial biopsy: a safe, well-tolerated and reliable technique for obtaining high-quality synovial tissue from both large and small joints in early arthritis patients. Ann Rheum Dis 2015;74:611-7.

15. Lazarou I, D’Agostino MA, Naredo E, Humby F, Filer A, Kelly SG Ultrasound-guided synovial biopsy: a systematic review according to the OMERACT filter and recommendations for minimal reporting standards in clinical studies. Rheumatology 2015;54:1867-75.

16. Cuppen BV, Welsing PM, Sprengers JJ, Bijlsma JW, Marijnissen AC, van Laar JM, et al. Personalized biological treatment for rheumatoid arthritis: a systematic review with a focus on clinical applicability. Rheumatology 2016;55:826-39.

17. Wijbrandts CA, Vergunst CE, Haringman JJ, Gerlag DM, Smeets TJ, Tak PP. Absence of changes in the number of synovial sublining macrophages after ineffective treatment for rheumatoid arthritis: implications for use of synovial sublining macrophages as a biomarker. Arthritis Rheum 2007;56:3869-71.

18. de Hair MJ, Harty LC, Gerlag DM, Pitzalis C, Veale DJ, Tak PP. Synovial tissue analysis for the discovery of diagnostic and prognostic biomarkers in patients with early arthritis. J Rheumatol 2011;38:2068-72.

19. van de Sande MG, Baeten DL. Immunopathology of synovitis: from histology to molecular pathways. Rheumatology 2016;55:599-606.

J Rheumatol 2016;43:2089-91; doi:10.3899/jrheum.161285 